\title{
SIRT1 as a Potential Biomarker for Obesity
}

\author{
Stephen SALIM $^{1}$, Felicia KARTAWIDJAJAPUTRA ${ }^{2, *}$ and Antonius SuWANTO ${ }^{1}$ \\ ${ }^{1}$ Faculty of Biotechnology, Atma Jaya Catholic University of Indonesia, Jalan Jendral Sudirman 51 \\ Jakarta Selatan, Jakarta, Indonesia 12930 \\ ${ }^{2}$ Nutrifood Research Center, PT Nutrifood Indonesia, Jalan Rawabali II No.3 Kawasan Industri Pulogadung \\ Jakarta Timur, Jakarta, Indonesia 13920
}

(Received June 22, 2019)

\begin{abstract}
Summary Asian people generally have thin figures; yet, they often suffer from hidden alarming metabolic conditions due to high visceral fat area (VFA). Therefore, it is crucial to have a biomarker to predict visceral obesity to prevent further complications. SIRT1, a NAD-dependent deacetylase gene, is responsible for upregulating lipolysis genes and is downregulated after acute high-fat meal consumption. However, the chronic exposure effect remains unknown. The purpose of this study was to assess the association between SIRT1 mRNA expression, fat intake, and visceral obesity in Indonesian population. Methods: This cross-sectional study involved 38 healthy subjects (20-30 y old, not suffering any chronic diseases or fever, not taking any medication or treatment, not smoking, not drinking alcohol frequently, not being pregnant, and not breastfeeding). Dietary patterns from 24-h food recall, physical activities fom international physical activity questionnaire (IPAQ), medical data from annual medical check-up, and body compositions were measured using InBody720 and compared with SIRT1 expression from peripheral blood mononuclear cells (PBMCs) samples. Results: Subjects with excessive percentage of body fat (PBF) had a significantly higher body mass index (BMI) (normal: 20.28 \pm 2.09 , excessive: 23.86 \pm 3.71 , $p=0.023$ ) and VFA (normal: 48.00 \pm 9.38 , excessive: $79.17 \pm 16.14, p=5 \times 10^{-5}$ ). The SIRT1 mRNA expression was significantly higher in subjects with excessive PBF (normal: $1 \pm 0.43$, excessive: $3.68 \pm 2.62, p=0.018)$ and positively correlated with $\operatorname{PBF}(\rho=0.376$, $p=0.045)$. Conclusion: SIRT1 acted as a potential marker for obesity in the evaluated population.
\end{abstract}

Key Words obesity, SIRT1, fat consumption, BMI, visceral fat area

Obesity is one of the leading public health issues in South East Asia. In 2016, the prevalence of obesity in adults reached $30.1 \%$ in Indonesia, $40.8 \%$ in Thailand, $41.4 \%$ in Singapore, and $50.2 \%$ in Malaysia. Obesity raises the risk of other diseases, such as cardiovascular diseases, diabetes, and hypertension. With the increasing prevalence and mortality rates in many regions, obesity is rapidly affecting people with diverse cultural and economic backgrounds (1).

Obesity occurs when calorie consumption is greater than energy expenditure. This condition causes the body to store excess energy as fat, consequently increasing body weight and adiposity. Factors affecting energy balance are genetics, diet, lifestyle, and gut microbiome. The interaction between lifestyle (nutrient intake and physical activity) and genetic strongly influences the development of obesity (2-4).

Obesity in Asian population is not as obvious as in Caucasians since Asians appear to have a distinct "thin outside fat inside" (TOFI) trait of obesity; in which they look slim and have normal BMI; yet, suffer from an unhealthy metabolic condition due to excessive visceral

\footnotetext{
*To whom correspondence should be addressed.

E-mail: felicia@nutrifood.co.id
}

fat accumulation (5). Visceral fat differs from subcutaneous fat; in which visceral fat produces more proinflammatory cytokines, such as tumor necrosis factor-alpha (TNF-alpha) and interleukin-6 (IL-6), and less adiponectin which could promote insulin resistance and atherosclerosis.

Visceral fat is located inside the internal organs, which makes it even more difficult to detect $(6,7)$. Computed tomography (CT), Magnetic resonance imaging (MRI), Dual-energy X-ray absorptiometry, and bioelectric impedance are commonly used tools in visceral obesity diagnosis; but these methods possess these following drawbacks: unnecessary radiation exposure, inconvenient procedure, expensive cost, and equipment unavailability (8). Therefore, it is crucial to have a specific blood-based biomarker to predict visceral obesity status. Blood-based biomarker is more preferred because it is convenient, non-invasive, and reflects the chronic effect of high-fat consumption towards key organs involved in energy homeostasis, such as liver and adipose (9).

Nutrigenomics is a branch of science which assesses the effects of nutrients on gene expression. This might be useful for developing effective dietary interventions to overcome obesity by using obesity biomarkers. Obesity biomarker refers to a gene transcript which will be 
upregulated or downregulated depending on the obesity status of an individual. SIRT1 encodes for Sirtuin 1 (SIRT1), a NAD-dependent protein deacetylase, which downregulates the expression of adipogenesis genes and upregulates the expression of lipolysis genes in response to different environmental stimuli, such as caloric restriction, fasting, caloric surplus, and excessive fat consumption (10).

An in vivo study in mice and monkey hearts showed that SIRT1 was significantly upregulated in response to oxidative stress (11). Oxidative stress is a common trait in obesity and the SIRT1 expression is predicted to increase in obese individuals to counteract $\mathrm{NAD}^{+}$depletion as well as mitochondrial dysfunction. Therefore, it is possible to examine the correlation between high-fat and low-fat diet and obesity risk by using this biomarker.

The previous human study has reported SIRT1 which is an anti-inflammatory gene to be downregulated after acute high-fat and high-calorie meal consumption. However, the effects of chronic high-fat and high-calorie diet on SIRT1 expression in human PBMCs are yet to be confirmed. Therefore, this dysregulation of gene expression profile might provide the insight of diet-induced obesity (12). PBMCs are suitable in the research of immune dysfunction in obesity since it is easily accessible and reflect the responses of dietary modifications and oxidative stress at the gene expression level (13).

Devising an effective strategy to combat this epidemic will require insight from both genetics and lifestyle. Despite the vital role of this interaction, the current research remains unexplored in Indonesian. Therefore, this study aimed to investigate the interaction between lifestyle (dietary intake and physical activity), SIRT1 gene expression, and other phenotype characteristics in Indonesian population.

\section{MATERIALS AND METHODS}

Subjects recruitment. Thirty-eight subjects (21 females and 17 males) were selected in this study, with the following criteria: aged between 20-30 y old, did not suffer any chronic diseases or fever, did not take any medication or treatment, did not smoke, did not drink alcohol frequently (five times or more in the past month), not being pregnant and not breastfeeding. The subjects were then classified into normal and excessive group based on their PBF. The cutoff points applied to this study were based on InBody720 (Biospace, Gangnam-gu, Seoul, Korea) Results Interpretation and Application Manual, in which PBF above 20\% and 28\% were considered as obese in male and female respectively.

Body composition analysis and medical data collection. Before participating in this study, the subjects had to undergo medical check-up procedure and body composition measurement. The body composition was measured using InBody720 (Biospace, Gangnam-gu, Seoul, Korea) which was based on bioelectrical impedance method. The instrument measured the total water value and low electric current resistance within the human body to predict the body fat mass and non-fat mass percentage (14). This instrument could also analyze other body composition such as BMI, waist-hip ratio (WHR), PBF, VFA, and basal metabolic rate (BMR). The medical data collected from subjects were triglyceride, total cholesterol, high-density lipoprotein (HDL), low-density lipoprotein (LDL), blood glucose, systolic and diastolic blood pressure.

Twenty-four-hour food recall and physical activity survey. All subjects were involved in 24-h food recall procedure to investigate their nutrient intake in the last $24 \mathrm{~h}$. Twenty-four-hour food recall was held three times; twice on the weekdays and once on the weekend. During 24-h food recall, subjects were required to memorize the type and amount of foods and drinks they had consumed for the last $24 \mathrm{~h}$ and informed them to the interviewer. Food models were used as a tool for facilitating the 24-h food recall. The 24-h food recall data were further processed using Food Processor SQL 10.1.1; ESHA, Salem, Oregon to obtain the detailed information of the nutrient intake.

The activity survey was conducted using the IPAQ. IPAQ calculated METS from the physical activity taken for the past seven days. The Metabolic equivalents (METs) were assigned to each activity based on the duration and intensity of activity. METs is defined as the amount of oxygen consumed when sitting at rest (1 METs $\left.=3.5 \mathrm{~mL} \mathrm{O} 2 \cdot \mathrm{kg}^{-1} \cdot \mathrm{min}^{-1}\right)$. The energy expended can be calculated by dividing the relative oxygen cost of the activity $(\mathrm{ml} \mathrm{O} / \mathrm{kg} / \mathrm{min})$ by 3,5 . The METs values of various activities were derived from the previous study by Ainsworth et al. in the compendium of physical activities (15). The subjects were classified into low, moderate, and high activity categories based on their METS and activity intensity (16).

SIRT1 expression analysis. Blood samples were collected from all subjects after $8 \mathrm{~h}$ of fasting. The mRNAs were isolated from whole blood using QIAamp ${ }^{\circledR}$ RNA Blood Mini Kit (Qiagen, Venlo, Limburg, Netherland). The isolated mRNA concentration was determined using NanoDrop 2000 (Thermo Scientific, Massachusetts, United States). The cDNA were then synthesized using RevertAid First Strand cDNA Synthesis Kit (Thermo Fisher Scientific, Waltham, Massachusetts, Amerika Serikat) and PCR GS 482 (G-Storm, Somerset, United Kingdom). The cDNA were amplified by SYBR green StepOnePlus Real-Time PCR System (Thermo Fisher Scientific, Waltham, Massachusetts, United States) using specific primers to analyze the gene expression. The SIRT1 primer set were obtained from Breitenstein et al. study (17). The PCR cycling conditions were initial denaturation at $95^{\circ} \mathrm{C}$ for $10 \mathrm{~min}$, followed by 40 cycles of denaturation at $95^{\circ} \mathrm{C}$ for $30 \mathrm{~s}$, annealing at $57^{\circ} \mathrm{C}$ for $1 \mathrm{~min}$, and extension at $72^{\circ} \mathrm{C}$ for $1 \mathrm{~min}$. The relative gene expressions were determined using Livak and Schmittgen 2- $\Delta \Delta \mathrm{CT}$.

Statistical analysis. Statistical analyses were performed with SPSS Statistics 21 (IBM, Armonk, New York, United States). The mean difference between groups was analyzed using Student's t-test. The strength 
Table 1. Characteristics of the study subjects according to PBF.

\begin{tabular}{lccc}
\hline \multicolumn{1}{c}{ Parameters } & $\begin{array}{c}\text { Normal PB } \\
(n=7)\end{array}$ & $\begin{array}{c}\text { Excessive PBF } \\
(n=22)\end{array}$ & $p$ value \\
\hline Age & $26.29 \pm 2.98$ & $26.64 \pm 2.30$ & 0.746 \\
Energy intake (kcals) & $1,751.74 \pm 472.56$ & $1,886.24 \pm 533.39$ & 0.556 \\
Energy from fat (\%) & $30.50 \pm 4.88$ & $35.34 \pm 5.69$ & 0.053 \\
BMI & $20.28 \pm 2.09$ & $23.86 \pm 3.71$ & $0.023^{\mathrm{a}}$ \\
WC $(\mathrm{cm})$ & $70.43 \pm 5.68$ & $77.59 \pm 11.57$ & 0.129 \\
WHR & $0.83 \pm 0.03$ & $0.87 \pm 0.05$ & 0.066 \\
VFA $\left.(\mathrm{cm})^{2}\right)$ & $48.00 \pm 9.38$ & $79.17 \pm 16.14$ & $5 \times 10^{-5 a}$ \\
PBF $(\%)$ & $22.19 \pm 6.11$ & $30.89 \pm 5.06$ & $0.002^{\mathrm{a}}$ \\
Total cholesterol (mg/dL) & $175.29 \pm 27.72$ & $178.82 \pm 28.84$ & 0.642 \\
LDL (mg/dL) & $109.43 \pm 20.58$ & $117.50 \pm 28.31$ & 0.493 \\
HDL (mg/dL) & $61.57 \pm 21.14$ & $54.82 \pm 10.08$ & 0.818 \\
Triglyceride (mg/dL) & $76.57 \pm 18.96$ & $95.77 \pm 40.92$ & 0.442 \\
METs/wk & $2,497.86 \pm 2,697.74$ & $1,198 \pm 1,457.17$ & 0.067 \\
SIRT1 expression & $1.00 \pm 0.43$ & $3.68 \pm 2.62$ & $0.018^{\mathrm{a}}$ \\
\hline
\end{tabular}

${ }^{\mathrm{a}} p \leq 0.05$ was considered statistically significant (two-tailed).

of association between nutrient intake, obesity parameter, and gene expression level were determined using Pearson product-moment correlation and Spearman's rank-order. All $p$ values reported in this study were twotailed and $p \leq 0.05$ were considered as statistically significant.

Statement of Ethics: The informed consent was obtained from all subjects according to the Helsinki Declaration. This study was reviewed and approved by Ethical Commissions of Atma Jaya Catholic University of Indonesia, approval number: 1468/III/LPPM-PM. 10.05/09/2016.

\section{RESULTS}

SIRT1 mRNA expression was upregulated by high-fat diet: The subjects were classified based on their PBF into two groups, normal $(n=7)$ and excessive $(n=22)$ (normal: 22.19 \pm 6.11 , excessive: 30.89 $\pm 5.06, p=0.002$ ). Then, obesity parameters were compared between the two groups. Compared to the normal group, the excessive group had statistically significant higher BMI (normal: 20.28 \pm 2.09 , excessive: $23.86 \pm 3.71, p=0.023$ )

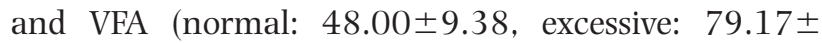
$\left.16.14, p=5 \times 10^{-5}\right)$. The excessive group also showed trends for higher waist-circumference (WC), WHR, total cholesterol, LDL, triglyceride, and lower HDL value compared with those in normal group (Table 1).

Our results also showed that the excessive PBF group had higher total fat intake (normal: $30.50 \pm 4.88$, excessive: $35.34 \pm 5.69, p=0.053$ ) and energy intake (normal: 1751.74 \pm 472.56 , excessive: $1886.24 \pm 533.39$, $p=0.556)$ compared to normal group, but the value was not statistically significant (Table 1 ).

Moreover, the expression of SIRT1 mRNA was also significantly elevated in individuals with excessive PBF (normal: $1 \pm 0.43$, excessive: 3.68 $\pm 2.62, p=0.018$ ) (Table 1). A significant correlation was found between SIRT1 and PBF $(\rho=0.376, p=0.045)$; whereas, no cor-
Table 2. Correlation between SIRT1 mRNA expression with fat intake and PBF.

\begin{tabular}{lcl}
\hline \multicolumn{1}{c}{ Parameters } & Spearman's Rho & $p$ value \\
\hline Energy from fat (\%) & 0.351 & 0.062 \\
PBF (\%) & 0.376 & $0.045^{\mathrm{a}}$ \\
\hline
\end{tabular}

${ }^{a} p \leq 0.05$ was considered statistically significant (twotailed).

relation between SIRT1 and fat intake was found (Table 2).

\section{DISCUSSION}

When comparing transcriptomic profiles between obese and lean groups, PBF classification was preferred in this study because obesity was originally defined as excessive fat accumulation. Therefore, PBF might be more reliable compared to BMI when assessing the actual health condition of the subjects. Our data suggested that higher fat and calorie intake in the excessive PBF group might have contributed to the greater value of VFA, which acted as a visceral obesity marker.

Multiple factors are involved in the obesity comorbidities, but SIRT1 gene is recently emerging as a prominent biomarker. The increased SIRT1 expression in excessive PBF group was in line with our hypothesis that excessive adiposity would upregulate SIRT1 to compensate for the excessive oxidative stress and prevent mitochondrial dysfunction. The hypoxia condition in the later stage of obesity might repress miR-199a (a microRNA which repressed SIRT1 expression in earlier stage), and resulted in increased SIRT1 expression to compensate the low SIRT1 activity in adipose tissue.

This compensatory mechanism exerted protective effects against mitochondrial dysfunction in obesity by 
synthesizing more SIRT1 to deacetylate PGC $1 \alpha$. PGC1 $\alpha$ deacetylation led to the induction of downstream pathways that upregulate mitochondrial biogenesis genes expressions $(10,11)$. SIRT1 might also deacetylate and activate $\operatorname{PPAR} \alpha$, thereby upregulating fatty acid metabolism genes expressions as a response to increased free fatty acid in excessive PBF group $(18,19)$. Another compelling evidence that supports this result was the upregulation of BRCA1 in obese subjects. BRCA1 is a widely studied tumor suppressor gene and SIRT1 positive regulator. Therefore, the increased SIRT1 expression is due to BRCA1 upregulation to decrease lipogenesis in obesity (20-22).

Results from Lee et al. proved otherwise; the VFA, as measured by CT, was negatively correlated with SIRT1 gene expression from PBMCs in healthy Koreans. This result contradicted with our findings and suggested that visceral obesity might decrease SIRT1 gene expression. The differences in the subject's criteria and VFA measurement method might contribute to this discrepancy. Lee et al. included smokers and alcoholic in their study, while we excluded subjects with those 2 characteristics. Besides, instead of CT method, we used bioelectrical impedance method to measure VFA in our subjects. Furthermore, study from Lee et al. could not correlate SIRT1 gene expression with food intake, since they did not assess subject's lifestyle as we did (23). This could be considered as an advance point, as our study could demonstrate a more holistic approach to evaluate potential factors influencing SIRT1 gene expression.

In our study, the positive correlation between fat intake, body fat percentages, and SIRT1 confirmed that higher fat consumption might increase the risk of obesity; and that SIRT1 is a potential marker for obesity. Unfortunately, it was not possible to assess the causal effect of high-fat diet, because the format for this study was not an intervention study. Since none of the previous PBMCs SIRT1 research had assessed the long-term consequences of high-fat diet and excessive fat accumulation, this finding would present a novel understanding of diet-induced obesity.

The limitations of this study were small sample size, lack of case-control group, and the absence of other biomarkers measurement. Further studies involving a larger sample size in the Indonesian population will be necessary to validate the result of this study. A case-control study to compare gene expressions profile and frequency of high-fat diet exposure between healthy or unhealthy individuals might be useful to determine the relationship between the high-fat diet and obesity. Additionally, obesity biomarkers, such as IL-6, TNF- $\alpha$, and adiponectin should also be measured to confirm this conclusion.

In conclusion, our study provided a novel finding that SIRT1 mRNA expression was significantly associated with visceral obesity in Indonesian population. However, further studies are required to ellaborate the mechanism behind the relation between SIRT1 and obesity.

\section{Disclosure of state of COI}

FK is an employee of PT. Nutrifood Indonesia. All other authors declared no competing interest.

\section{REFERENCES}

1) World Health Organization (WHO). 2016. Diabetes country profiles 2016. Available from: https://www. who.int/diabetes/country-profiles/en/(updated 2017 May 26).

2) Hall KD, Heymsfield SB, Kemnitz JW, Klein S, Schoeller DA, Speakman JR. 2012. Energy balance and its components: implications for body weight regulation. Am J Clin Nutr 95(4): 989-994.

3) Hill JO, Wyatt HR, Peters JC. 2012. Energy balance and obesity. Circulation 126(1): 126-132.

4) Parekh PJ, Balart LA, Johnson DA. 2015. The influence of the gut microbiome on obesity, metabolic syndrome and gastrointestinal disease. Clin Transl Gastroenterol 6: e91.

5) Leow MKS. 2017. Characterization of the Asian phenotype-an emerging paradigm with clinicopathological and human research implications. Int J Med Sci 14(7): 639-647.

6) Hamdy O, Porramatikul S, Al-Ozairi E. 2006. Metabolic obesity: the paradox between visceral and subcutaneous fat. Curr Diabetes Rev 2(4): 367-373.

7) Ye S, Wen H, Jiang Z, Wu X. 2016. The effect of visceral obesity on clinicopathological features in patients with endometrial cancer: a retrospective analysis of $200 \mathrm{Chi}-$ nese patients. BMC Cancer 16: 209.

8) Snijder MB, Visser M, Dekker JM, Seidel JCl, Fuerst T, Tylavsky F, Cauley J, Lang T, Nevitt M, Harris TB. 2002. The prediction of visceral fat by dual-energy X-ray absorptiometry in the elderly: a comparison with computed tomography and anthropometry. Int J Obes 26(7): 984-993.

9) Lau FC, Bagchi M, Sen C, Roy S, Bagchi D. 2008. Nutrigenomic analysis of diet-gene interactions on functional supplements for weight management. Curr Genomics 9(4): 239-251.

10) Houtkooper RH, Pirinen E, Auwerx J. 2012. Sirtuins as regulators of metabolism and health span. Nat Rev Mol Cell Biol 13(4): 225-238.

11) Alcendor RR, Gao S, Zhai P, Zablocki D, Holle E, Yu X, Tian B, Wagner T, Vatner SF, Sadoshima J. 2007. SIRT1 regulates aging and resistance to oxidative stress in the heart. Circ Res 100(10): 1512-1521.

12) Sampson JNB. 2017. A high-fat meal alters post-prandial mRNA expression of SIRT1, SIRT4, and SIRT6. Available from: https://digital.library.unt.edu/ark:/67531/ metadc822825/m1/1/(updated 2017 May 26).

13) Catalán V, Gómez-Ambrosi J, Rodríguez A, Ramírez B, Valentí V, Moncada R, Silva C, Salvador J, Frühbeck G. 2015. Peripheral mononuclear blood cells contribute to the obesity-associated inflammatory state independently of glycemic status: involvement of the novel proinflammatory adipokines chemerin, chitinase-3-like protein 1 , lipocalin-2 and osteopontin. Genes Nutr 10(3): 460.

14) Beechy L, Galpern J, Petrone A, Das SK. 2012. Assessment tools in obesity-Psychological measures, diet, activity, and body composition. Physiol Behav 106(7): 154-171.

15) Ainsworth BE1, Haskell WL, Herrmann SD, Meckes N, Bassett DR Jr, Tudor-Locke C, Greer JL, Vezina J, WhittGlover MC, Leon AS. 2011. 2011 compendium of phys- 
ical activities: A second update of codes and MET values. Med Sci Sports Exerc 43(8): 1575-1581.

16) Craig CL, Marshall A, Sjostrom M, Bauman AE, Booth ML, Ainsworth BE, Pratt M, Ekelund U, Yngve A, Sallis JF, Oja P. 2003. International Physical Activity Questionnaire: 12 country reliability and validity. Med Sci Sports Exerc 35(8): 1381-1395.

17) Breitenstein A, Wyss CA, Spescha RD, Franzeck FC, Hof D, Riwanto M, Hasun M, Akhmedov A, von Eckardstein A, Maier W, Landmesser U, Lüscher TF, Camici GG. 2013. Peripheral blood monocyte SIRT1 expression is reduced in patients with coronary artery disease. PLoS One 8(1): e 53106.

18) Bonen A, Tandon NN, Glatz JF, Luiken JJ, Heigenhauser GJ. 2006. The fatty acid transporter FAT/CD36 is upregulated in subcutaneous and visceral adipose tissues in human obesity and type 2 diabetes. Int J Obes 30(6): 877-883.
19) Serra D, Mera P, Malandrino MI, Mir JF, Herrero L. 2013. Mitochondrial fatty acid oxidation in obesity. Antioxid Redox Signal 19(3): 269-284.

20) Ortega FJ, Moreno-Navarrete JM, Mayas D, García-Santos E, Gómez-Serrano M, Rodriguez-Hermosa JI, Ruiz B, Ricart W, Tinahones FJ, Frühbeck G, Peral B, and Fernández-Real JM. 2012. Breast cancer 1 (brca1) may be behind decreased lipogenesis in adipose tissue from obese subjects. PLoS One 7(5): e33233.

21) Li D, Bi FF, Chen NN, Cao JM, Sun WP, Zhou YM, Li CY, Yang Q. 2014. A novel crosstalk between brca1 and sirtuin 1 in ovarian cancer. Sci Rep 4: 6666.

22) Wang XX, Li D. 2015. Linking brca1 to NAD world. Cell Cycle 14(7): 941-942.

23) Lee H, Chu SH, Park JY, Park HK, Im JA, Lee JW. 2013. Visceral adiposity is associated with SIRT1 expression in peripheral blood mononuclear cells: a pilot study. Endocr J 60(11): 1269-1273. 